D.O.I.: $10.3895 / \mathrm{S} 1808-04482010000300001$

\title{
AVALIAÇÃO DA OPERAÇÃO DE SETUP EM UMA CÉLULA DE MANUFATURA DE UMA INDÚSTRIA DE AUTOPEÇAS ATRAVÉS DA SIMULAÇÃO A EVENTOS DISCRETOS
}

\section{EVALUATION OF SETUP OPERATION IN A MANUFACTURE AUTO PARTS CELL THROUGH DISCRETE EVENTS SIMULATION}

\author{
Rafael de Carvalho Miranda ${ }^{1}$; Bárbara Coutinho Fernandes ${ }^{2}$; Janaína Rodrigues Ribeiro ${ }^{3}$; José \\ Arnaldo Barra Montevechi ${ }^{4}$; Alexandre Ferreira de Pinho \\ ${ }^{1}$ Universidade Federal de Itajubá - UNIFEI - Itajubá - Brasil \\ mirandaprod@yahoo.com.br \\ ${ }^{2}$ Universidade Federal de Itajubá - UNIFEI - Itajubá - Brasil \\ babifernan@yahoo.com.br \\ ${ }^{3}$ Universidade Federal de Itajubá - UNIFEI - Itajubá - Brasil \\ janaina_epr@yahoo.com.br \\ ${ }^{4}$ Universidade Federal de Itajubá - UNIFEI - Itajubá - Brasil \\ montevechi@unifei.edu.br \\ ${ }^{5}$ Universidade Federal de Itajubá - UNIFEI - Itajubá - Brasil \\ pinho@unifei.edu.br
}

\begin{abstract}
Resumo
Os ambientes de manufatura têm se tornado cada vez mais complexos e consequentemente os processos de tomada de decisão vêm adquirindo complexidade na mesma proporção. Neste contexto a simulação computacional tem se mostrado uma valiosa ferramenta capaz de ajudar na melhoria de processos e análise de mudanças. Este trabalho buscou por meio de um projeto de simulação computacional em uma célula de manufatura de uma empresa fornecedora de autopeças, determinar a melhor forma de execução de setup: dedicado ou em etapas. Concluiu-se que independentemente da forma como o setup era realizado, dedicado ou em etapas, a produção média da célula era estatisticamente igual. Sendo assim, como o setup dedicado era de execução mais fácil, outros três cenários foram criados a partir deste visando à otimização da operação de setup e aumento de produtividade da célula. Estes cenários foram construídos em acordo com os tomadores de decisão da empresa para que assim pudessem escolher o que melhor atendesse as suas necessidades.
\end{abstract}

Palavras-chave: simulação computacional; setup; manufatura.

\section{Introdução}

A constante evolução dos sistemas de produção, melhoria de qualidade dos produtos associados a uma concorrência cada vez mais acirrada exige por parte dos gestores que medidas sejam tomadas para garantir a sobrevivência de suas empresas no mercado. Adicionalmente, com o advento da globalização essa exigência crescente pela melhoria contínua de produtos e serviços se 
acelerou, de tal forma que empresas que não se adéquem a esta nova realidade estarão fadadas ao fracasso. Contudo nem sempre é aconselhável, fácil ou até mesmo possível promover alterações em linhas de produção complexas sem que se tenha certeza de que a alteração proposta é viável e trará benefícios a cadeia produtiva.

Neste contexto se insere a simulação a eventos discretos. A simulação permite avaliar e analisar sistemas reais a partir da construção de modelos computacionais por meio dos quais se pode responder a perguntas do tipo "o que ocorre se", tornando-se assim uma poderosa ferramenta de apoio a tomada de decisão (CHWIF; MEDINA, 2007).

No passado, a simulação era considerada uma técnica de último recurso, que deveria ser utilizada quando todas as técnicas possíveis falhassem (LAW; KELTON, 2000). Os mesmos autores afirmam que com o passar do tempo, no entanto, a simulação vem se tornando uma das técnicas mais utilizadas, como podem comprovar as pesquisas de campo. Ryan e Heavey (2006) apontam a simulação como uma das técnicas de pesquisa mais utilizadas devido à sua versatilidade, flexibilidade e poder de análise.

Sendo assim, conhecidos os benefícios no uso da simulação como suporte à tomada de decisão frente à análise de processos, o objetivo deste trabalho é desenvolver um projeto de simulação com a intenção de responder qual o melhor método de trabalho para a realização de setup (preparação) em uma célula de manufatura de uma empresa de autopeças do sul de Minas Gerais.

Segundo Barros e Moccellin (2004) para os casos em que os tempos de setup são significativos quando comparados com os tempos de processamento, há a necessidade de tratá-los de forma diferenciada, uma vez que eles têm relação direta com a disponibilidade de equipamentos e operadores, acarretando em custos específicos e influenciando de forma direta na produtividade.

Desta forma, este trabalho encontra-se estruturado em seis seções. A seção 2 apresenta uma breve fundamentação teórica abrangendo a simulação a eventos discretos e o impacto dos tempos de setup em um ambiente produtivo. Em seguida, a seção 3 apresenta a metodologia de pesquisa empregada. A seção 4 discute o desdobramento da metodologia e finalmente as seções 5 e 6 discutem os resultados alcançados, bem como as conclusões gerais deste trabalho.

\section{Referencial Teórico}

\subsection{A Simulação a eventos discretos}

Segundo Banks (1998), a simulação é a imitação da operação de um processo ou sistema do mundo real em uma base de tempo. A simulação envolve a geração de uma história artificial de um sistema e a observação desta história artificial, para a elaboração de inferências a respeito das características operacionais do sistema real que será representado. Já para Harrel, Ghosh e Bowden 
(2004) a simulação pode ser definida como a imitação de um sistema dinâmico usando um modelo computacional para avaliar e melhorar o desempenho deste sistema.

De acordo com Chwif e Medina (2007), os sistemas reais geralmente apresentam uma maior complexidade devido a sua natureza dinâmica e aleatória. Modelos de simulação conseguem capturar com mais fidelidade essas características, procurando repetir em um computador o mesmo comportamento que o sistema apresentaria quando submetido às mesmas condições de contorno. $\mathrm{O}$ modelo de simulação é utilizado, particularmente, como uma ferramenta para se obter respostas a sentenças do tipo: "o que ocorre se...”.

De acordo com Law (2009) um modelo de simulação é uma alternativa à experimentação direta com o sistema real (existente ou proposto). Uma vez que a experimentação direta geralmente propicia a interrupção do funcionamento do sistema produtivo, é inviável, ou é simplesmente impossível de ser realizada.

Autores como Banks et al. (2005), O’kane, Spenceley, e Taylor (2000) afirmam que a simulação tem se tornado uma das técnicas mais populares para se analisar problemas complexos em ambientes de manufatura.

Segundo Kelton (1999), o uso da simulação vem auxiliar diretamente a execução de experimentos custosos ou mesmo impossíveis de serem realizados na prática.

Com a evolução dos estudos na área, a simulação a eventos discretos tem sido cada vez mais utilizada e os benefícios com ela obtidos têm gerado impactos nos mais diferentes sistemas (serviços, hospitais, manufatura, militar e logística).

A literatura traz inúmeros exemplos de sua aplicação, tais como: no estudo de Lam e Lau (2004) a simulação foi utilizada para comprovar a viabilidade das propostas de reestruturação de um call-center, no estudo de Abdulmalek e Rajgopal (2007) foi feita uma análise dos benefícios do Lean Manufacturing e VSM (Value Stream Mapping) através da simulação computacional. No estudo de Ekren et al. (2010) a simulação foi utilizada junto com o planejamento de experimentos para identificação dos fatores que mais impactam em um ambiente produtivo. No trabalho de Oliveira et al. (2006) a simulação foi utilizada na avaliação do impacto do DFA (Design for Assembly). Já Ahmed e Alkhamis (2009) utilizaram a simulação no setor de saúde para otimizar o setor de emergência num hospital do Kuwait.

Algumas etapas para a simulação de sistemas são propostas por alguns autores. Chwif (1999) propõe em seu trabalho um ciclo envolvendo a concepção, implementação e análise, como mostra a Figura 1. 
Figura 1 - Seqüência de passos para a simulação

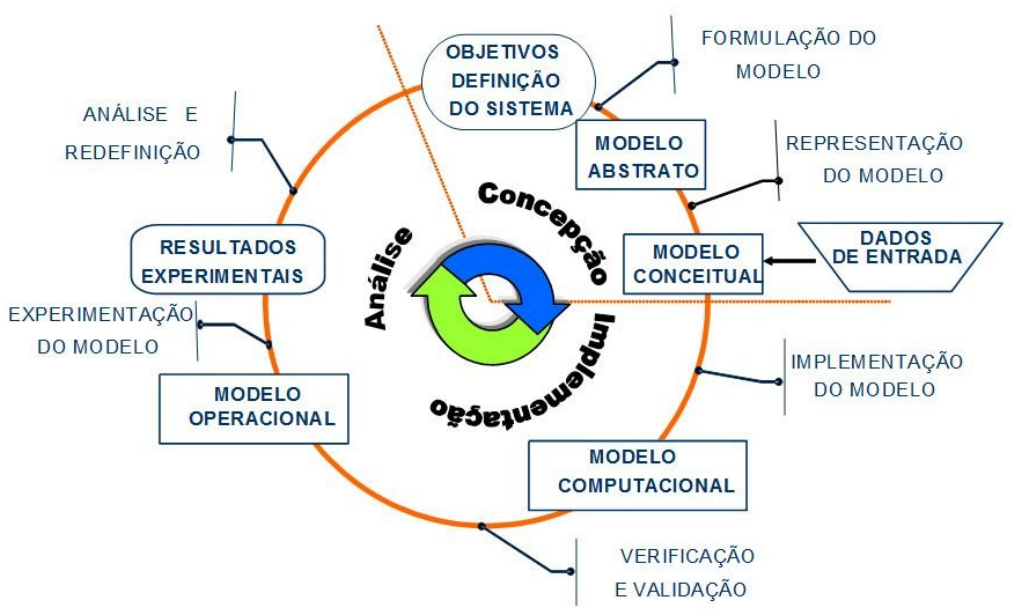

Fonte: Chwif (1999)

Segundo Paiva et al. (2009), na fase de concepção o analista de simulação deve entender claramente o sistema a ser simulado e os seus objetivos, através da discussão do problema com especialistas. Os dados de entrada também são coletados nesta fase. Ainda na etapa de concepção, o modelo que está na mente do analista (modelo abstrato) deve ser representado de acordo com alguma técnica de representação de modelos de simulação, a fim de torná-lo um modelo conceitual.

Na fase de implementação do modelo, o modelo conceitual é convertido em um modelo computacional através da utilização de alguma linguagem de simulação ou de um simulador comercial. O modelo computacional implementado deve ser comparado ao modelo conceitual, com a finalidade de avaliar se a sua operação atende ao que foi estabelecido na etapa de concepção. Alguns resultados devem ser gerados para a validação do modelo computacional, observando se o modelo é uma representação precisa da realidade (PAIVA et al., 2009).

Ainda segundo os mesmos autores, na terceira fase, análise dos resultados, o modelo computacional está pronto para a realização de experimentos, dando origem ao modelo experimental ou modelo operacional. Nesta etapa são efetuadas várias rodadas do modelo e os resultados da simulação são analisados e documentados. A partir dos resultados, conclusões e recomendações sobre o sistema podem ser geradas. Caso necessário, o modelo pode ser modificado, e este ciclo é reiniciado.

\subsection{O impacto dos tempos de setup em um ambiente produtivo}

Segundo Slack, Chambers, e Johnston (2002) o tempo de setup pode ser definido como o tempo decorrido na troca do processo da produção de um lote até a produção da primeira peça boa do próximo lote. 
Grande parte das pesquisas em programação consideram os tempos de setup como não relevantes ou de pequena variabilidade incorporando-os na maioria das vezes, no próprio tempo de processamento (ALLAHVERDI; GUPTA; ALDOWAISAN, 1999).

Esse procedimento simplifica muito a análise em determinadas aplicações, principalmente quando os tempos de setup são consideravelmente menores que os tempos de processamento, ou em casos em que o setup é executado uma única vez. No entanto, para os casos em que os tempos de setup apresentam razão significativa diante dos tempos de processamento, há a necessidade de tratálos diferenciadamente, uma vez que eles têm relação direta com a disponibilidade de equipamentos e acarretam em custos específicos (BARROS; MOCCELLIN, 2004).

Kim e Bobrowski (1994) definem assim a importância do tempo de setup:

- O tempo de setup afeta diretamente a taxa de saída do sistema de produção;

- O custo de realização do setup geralmente é mais alto que o custo de processamento, visto que envolve outros fatores como o custo de máquina parada e o custo de mão-de-obra de especializada;

- O setup pode ser executado por pessoal especializado, que pode vir a ser um recurso limitado e nem sempre disponível.

- Harmon e Peterson (1991) apresentam três razões chaves para redução do tempo gasto na realização do setup:

- Se o custo de setup é alto, os lotes de fabricação tendem a serem grandes, aumentando assim os investimentos em estoques;

- As técnicas de troca de ferramentas mais rápidas e simples diminuem a possibilidade de erros na regulagem dos equipamentos;

- A redução do tempo de setup resulta em aumento do tempo de operação do equipamento.

Desta forma o setup pode ser visto como relevante no processo produtivo. Sua redução diminui a necessidade de pessoal para sua execução e, simultaneamente, aumenta o tempo disponível da máquina. Além disso, proporciona reduções de estoque em processo, de lead time e de processamento (BARROS, 2002; SLACK; CHAMBERS; JOHNSTON, 2002).

\section{Metodologia de pesquisa}

O trabalho aqui desenvolvido segue o método de modelagem e simulação. Este método segundo Chung (2004) é o processo de criar e experimentar um sistema físico através de um modelo matemático computadorizado. Um sistema pode ser definido como um conjunto de componentes ou processos que se interagem e que recebem entradas e oferecem resultados para algum propósito. 
Figura 2 - Fluxograma da metodologia de pesquisa



Fonte: Montevechi et al. (2010)

O método de pesquisa utilizado neste trabalho foi adaptado do trabalho de Montevechi et al. (2010). Segundo este método três modelos deverão ser elaborados em três etapas distintas: o modelo conceitual na etapa de concepção, o computacional na etapa de implementação e finalmente o modelo operacional na etapa de análise.

Para melhor compreensão, a figura 2, apresenta passo a passo a metodologia utilizada, adaptada do trabalho de Montevechi et al. (2010). Cada etapa do método de pesquisa apresentada na 
figura 2 será detalhada na próxima sessão, juntamente com sua aplicação.

\section{Aplicação da metodologia}

\subsection{Concepção}

A primeira etapa a ser trabalhada segundo o método de pesquisa consiste na definição dos objetivos do estudo, construção e validação do modelo conceitual e modelagem dos dados de entrada.

\subsubsection{Objetivos e definição do sistema}

A célula de manufatura onde o projeto de simulação foi realizado passava por um processo de implementação da filosofia Lean Manufacturing. A linha de produção em questão segue um fluxo de operações que vão desde o recebimento da matéria prima até a inspeção final e embalagem dos produtos, tudo realizado sequencialmente dentro dos princípios da manufatura enxuta. Devido à solicitação de confidencialidade por parte da empresa este fluxo de operações será omitido.

As atividades do início da cadeia produtiva (torneamento e fresamento) foram escolhidas pela gerência para o estudo de simulação devido a apresentarem elevados tempos de setup, com mais de 36 horas mensais como pode ser visto na figura 3, e a indefinição do melhor método de trabalho para sua realização.

Figura 3 - Perdas mensais registradas na célula sob estudo

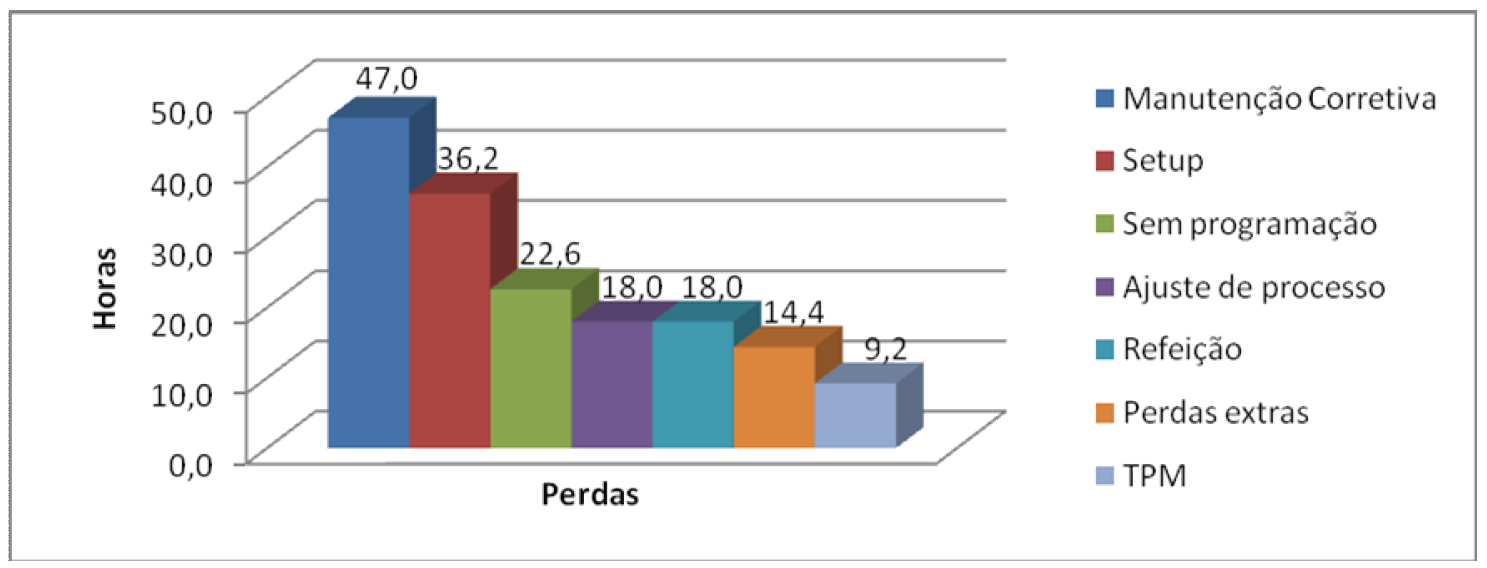

Fonte: Autoria própria (2010)

Sendo assim, o objetivo desse trabalho consistiu em desenvolver um projeto de simulação computacional, em uma célula de manufatura de uma empresa fornecedora de autopeças, de forma a determinar a melhor forma de execução do setup das operações de torneamento e fresamento.

Inicialmente este trabalho buscou responder a empresa qual a forma de setup conduziria a uma produtividade maior: o setup realizado em etapas, como era realizado antes do projeto de 
simulação e determinado pela gerência ou de forma dedicada, de realização mais simples e preferido pelos operadores. Uma vez respondida tal questão, cenários foram gerados visando incrementar a produtividade no método de trabalho escolhido.

\subsubsection{Construção e validação do modelo conceitual}

O mapeamento das atividades a serem simuladas foi o ponto de partida desta etapa, e forneceu informações essenciais para elaboração do modelo computacional. De acordo com Ryan e Heavey (2006) existem diversas técnicas de mapeamento de processos, entretanto poucas delas oferecem suporte necessário à construção de um modelo computacional.

Para este trabalho, foi utilizada a técnica de fluxograma. Fitzsimmons e Fitzsimmons (2000) definem esta técnica como uma representação visual de processos onde podem ser registrados, além das atividades e informações, os pontos de tomada de decisão. Além disso, é uma ferramenta amplamente conhecida e de fácil entendimento por todos os níveis operacionais, o que torna o processo de validação do modelo mais ágil junto a especialistas.

O processo analisado é composto por duas bancadas, duas máquinas, e uma área de inspeção. Antes de ser processada, a matéria-prima deve ser preparada em uma das bancadas. Uma vez preparada, a matéria-prima agora em forma de pacote, pode ser processada pelas máquinas. Cada uma das máquinas realiza funções de torneamento e fresagem independentemente, ou seja, as operações de torneamento e fresagem podem ocorrer simultaneamente e possuem tempos de processamento diferentes. Os pacotes são primeiramente torneados e em seguida passam para a etapa de fresamento. Todo o processo descrito (preparação, operação e inspeção) acontece para as duas máquinas e é realizado por apenas um único operador, responsável também pelo setup das duas máquinas.

As figuras 4 e 5 apresentam o modelo conceitual desenvolvido. Vale ressaltar que a figura 4 representa as atividades que ocorrem em uma das máquinas da célula, no entanto este fluxograma é válido para ambas as máquinas pertencentes ao sistema uma vez que estas são iguais. A figura 5 apresenta o fluxograma isolado da atividade de setup, realizado em uma das máquinas, simultaneamente a operação da outra. Devido a seu caráter técnico, as etapas de setup no fluxograma da figura 5 foram simplificadas. 
Figura 4 - Modelo conceitual - Fluxograma da operação da célula



Fonte: Autoria própria (2010)

Figura 5 - Modelo conceitual - Fluxograma simplificado da atividade de setup

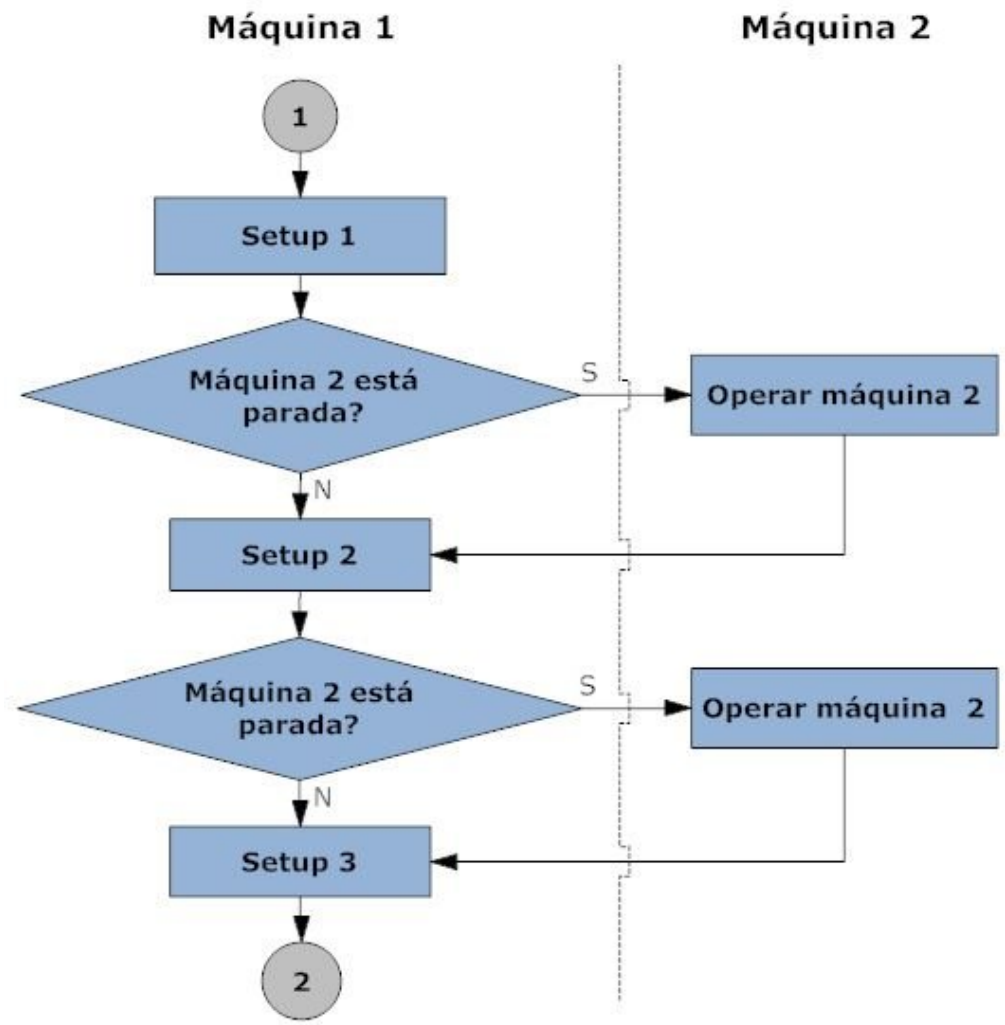

Fonte: Autoria própria (2010) 
Ao final da elaboração do modelo conceitual, o mesmo foi validado através da técnica de validação face a face. Técnica segundo a qual os especialistas no sistema compreendem o modelo como correto (SARGENT, 2008). Com o modelo conceitual validado passou-se para a etapa seguinte.

\subsubsection{Modelagem dos dados de entrada}

Esta etapa é composta por três passos: coleta dos dados, tratamento dos dados e inferência.

Foram registradas quinze tomadas de tempo para cada operação realizada pelo operador. Estes dados foram dispostos no software Minitab ${ }^{\circledR}$ para tratamento dos dados e inferência.

O teste de identificação de distribuição foi utilizado apontando a melhor distribuição dos dados. Os resultados obtidos, bem como o melhor tipo de distribuição obtido através do Minitab® podem ser vistos na tabela 1 .

Tabela 1 - Tempos de processamento do operador

\begin{tabular}{cc}
\hline Processo & Tempo de operação (s) \\
\hline Preparação 1 & Normal $(28.1,2.3)$ \\
Torneamento 1 & Normal $(10,1.1)$ \\
Fresagem 1 & Normal $(10,1.1)$ \\
Acabamento 1 & Normal $(18,1.91)$ \\
Preparação 2 & Normal $(28.1,2.9)$ \\
Torneamento 2 & Normal $(10,1.1)$ \\
Fresagem 2 & Normal $(10,1.1)$ \\
Acabamento 2 & Normal $(18,1.91)$ \\
Inspeção & Normal $(60,12)$ \\
\hline
\end{tabular}

Fonte: Pesquisa de campo (2010)

Com relação ao tempo de processamento das máquinas este foi fornecido pela empresa. Devido a normas de seguranças internas apenas a equipe de tempos e métodos da empresa poderiam colhê-los. Estes podem ser observados na tabela 2.

Tabela 2 - Tempos de processamento da máquina

\begin{tabular}{ccc}
\hline Processo & Tempo de processamento (min) \\
\hline Torno 1 & Normal $(3.1,0.62)$ \\
Fresadora 1 & Normal $(2.2,0.44)$ \\
Torno 2 & Normal $(3.1,0.62)$ \\
Fresadora 2 & Normal $(2.2,0.44)$ \\
\hline
\end{tabular}

Fonte: Pesquisa de campo (2010) 


\subsection{Implementação}

A fase de implementação teve início com a construção, verificação e validação do modelo computacional.

\subsubsection{Modelo computacional: construção e verificação}

Para a construção do modelo computacional foi utilizado o simulador Promodel ${ }^{\circledR}$. Tal escolha se deve à experiência dos autores com modelagens neste simulador e pelo software apresentar características de animação que auxiliam no processo de verificação e validação do modelo computacional.

Foram desenvolvidos seis modelos em ordem crescente de complexidade, e ao final de cada modelo seus erros foram depurados e sua lógica corrigida.

Foram definidas as "entidades" (peças produzidas) e as rotas que estas e o "recurso" (operador) iriam percorrer. A tabela 3 apresenta a lógica de entrada e saída das entidades no sistema, além de apresentar os locais onde as mesmas seriam processadas. Na figura 6 vê-se a tela do software com o layout para o modelo simulado.

Tabela 3 - Descrição: Processo - Entradas x Saídas

\begin{tabular}{ccc}
\hline Processo & Entradas & Saídas \\
\hline Bancada & Matéria prima & Pacote \\
Torno & Pacote & Pacote \\
Fresadora & Pacote & Pacote usinado \\
Inspeção & Pacote usinado & Pacote inspecionado \\
\hline
\end{tabular}

Fonte: Pesquisa de campo (2010)

Figura 6 - Tela do Promodel® para o modelo simulado

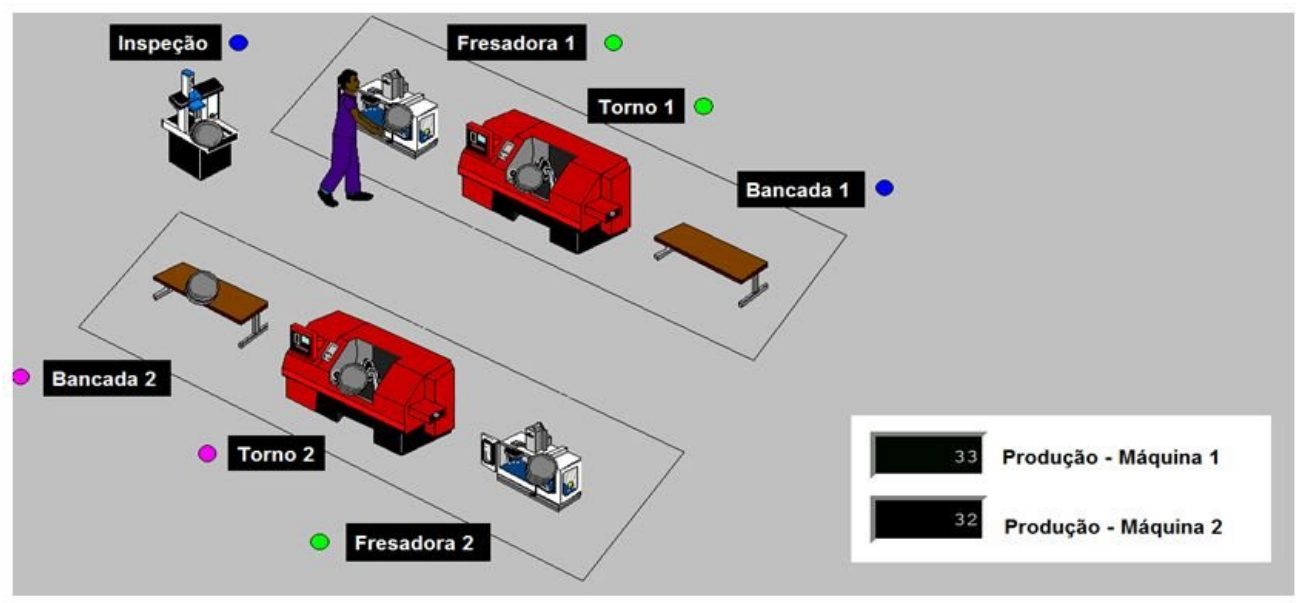

Fonte: Autoria própria (2010) 
Após a construção do modelo computacional o mesmo foi verificado. Segundo Sargent (2008), a verificação de um modelo é definida ao assegurar-se que o programa computacional e sua implementação estão corretos. Neste modelo utilizou-se de contadores, variáveis e da animação gráfica para constatar possíveis erros na lógica do modelo.

\subsubsection{Validação do modelo computacional}

Segundo Sargent (2008), a validação do modelo computacional é definida como a determinação de que o comportamento do modelo simulado detém precisão suficiente para representar o modelo real para a aplicabilidade a qual se destina. Desta forma se o modelo não é uma aproximação bastante próxima do sistema real, todas as conclusões derivadas deste estarão susceptíveis a erros e poderão resultar em decisões incorretas. Sendo assim a validação pode e deve ser feita para todos os modelos, independentemente do sistema modelado (LAW, 2006).

A validação do modelo foi feita a partir da comparação entre dados do sistema real com os resultados obtidos pelo modelo. Para tal utilizou-se do teste de hipótese descrito por Triola (2005). Este teste permite comparar uma amostra em que se conhece a média e o desvio padrão (resultados simulados), com um único valor (média da população) com desvio padrão desconhecido (sistema real).

Este teste foi utilizado devido à ausência de dados reais fornecidos pela empresa, a qual forneceu apenas uma média semanal da célula de produção, inviabilizando a realização de outros testes de hipóteses ou outras formas de validação estatística.

Segundo dados históricos fornecidos pela empresa a célula de produção em questão produz em média 1317 produtos por semana.

De posse desse dado e dos dados provenientes de relatórios gerados pelo Promodel ${ }^{\circledR}$ para 10 réplicas pode-se realizar o teste de hipóteses descrito por Triola (2005) com auxílio do Minitab® (Tabelas 4 e 5).

Tabela 4 - Dados de entrada para realização do teste de hipóteses One-Sample T

\begin{tabular}{ccccc}
\hline Tamanho da amostra & Média & Desvio padrão & Média testada & Nível de confiança \\
\hline 10 & 1319 & 4,03 & 1317 & $95 \%$ \\
\hline Fonte: Autoria própria $(2010)$ & & &
\end{tabular}

Fonte: Autoria própria (2010)

Como o valor do $p$-value do teste realizado $(0,151)$ é superior ao nível de significância adotado $(\alpha=0,05)$, aceita-se a hipótese nula, ou seja a média do sistema simulado é igual a média do sistema real. Dessa forma aceita-se o modelo validado estatisticamente. Uma vez validado o modelo computacional partiu-se para a etapa de construção de cenários. 
Tabela 5 - Resultado do teste de hipóteses One-Sample T

\begin{tabular}{cccccccc}
\hline $\begin{array}{c}\text { Tamanho } \\
\text { da amostra }\end{array}$ & Média & $\begin{array}{c}\text { Desvio } \\
\text { padrão }\end{array}$ & $\begin{array}{c}\text { Erro da } \\
\text { média }\end{array}$ & $\begin{array}{c}\text { Intervalo de Confiança } \\
\mathbf{( 9 5 \% )}\end{array}$ & T-Value & P-Value \\
\hline 10 & 1319 & 4,03 & 1,27 & 1316,12 & 1321,88 & 1,57 & 0,151 \\
\hline Fon
\end{tabular}

Fonte: Autoria própria (2010)

\subsection{Análise}

Por fim a fase de análise trabalhará com o modelo operacional, execução de experimentos, análise dos resultados e conclusões.

Sanchez (2007) afirma que o processo de construir, verificar e validar um modelo de simulação pode ser dificil e longo, porém uma vez realizada tais etapas, então é o momento de ter o modelo trabalhando para o modelador.

\subsubsection{Criação dos cenários}

Nesta seção buscou-se apresentar primeiramente o cenário atual, onde o setup é realizado em etapas e o cenário 1, realizado de forma dedicada. Após a análise estatística realizada entre esses dois cenários, concluiu-se que estatisticamente não há diferença entre eles. Sendo assim como o setup dedicado é mais fácil de ser executado, a gerência optou por simular as melhorias propostas tendo como base este cenário. Isto justifica a criação dos cenários tendo como base apenas o cenário onde o setup era realizado de forma dedicado, abandonando a execução do setup em etapas.

\subsubsection{Cenário atual: setup em etapas}

O cenário atual conta com um único operador desempenhando as funções de operação, setup e inspeção de duas máquinas. Neste cenário quando ocorre mudança de lote, o operador se divide entre as funções de operação da máquina e realização de setup da outra máquina, ou seja, o operador desempenha as funções de setup de forma não dedicada, realizando-o em etapas e em paralelo com a operação da outra máquina.

\subsubsection{Cenário 1: setup dedicado}

Neste cenário o setup ocorrerá de forma dedicada. Assim, no momento de troca de lotes o operador dedica-se totalmente as atividades de setup abandonado as funções de operação da outra máquina, deixando-a nesse período ociosa. Vale ressaltar que o operador demonstrou aos pesquisadores interesse em adotar este método de produção, contrariando a determinação da gerência pelo cenário atual. 


\subsubsection{Cenário 2: máquina otimizada}

Este cenário foi sugerido pela gerência com o intuito de obter uma ferramenta para auxílio à tomada de decisão. Isso porque a empresa tem a intenção de substituir parte das máquinas da célula em estudo, com a finalidade de reduzir seus tempos de setup. No entanto a empresa não possui nenhum estudo relativo ao impacto desta melhoria para a produtividade que justifique sua adoção. Assim, o objetivo deste cenário é embasar a decisão da gerência em adotar ou não a troca de partes das máquinas do sistema para redução do tempo de setup. Com essa troca de maquinário a empresa visa reduzir o tempo de setup de 53,6 minutos para 38,45 minutos, o que representaria uma redução de $28 \%$. Neste cenário o setup foi realizado de forma dedicada uma vez que esse foi o método que a empresa optou por otimizar.

\subsubsection{Cenário 3: inclusão de um novo recurso}

Este é mais um cenário sugerido pela empresa com o intuito de obter uma ferramenta para auxílio à tomada de decisão. Neste cenário incluiu-se um novo recurso que possui uma única atividade: realizar o setup no momento de troca de lotes. Desta maneira o operador que já estava presente nos cenários anteriores poderia se dedicar somente as atividades de produção, não interferindo na operação de setup, que seria realizado por um operador especializado. Este operador adicional seria responsável pela realização dos setups de todas as operações da célula, e não apenas das atividades de torneamento e fresamento, justificando assim sua inclusão.

\subsubsection{Cenário 4: cenário ótimo}

Este cenário buscou otimizar todas as condições. O setup foi realizado de forma dedicada, com a máquina otimizada e com inclusão de um novo recurso responsável pela atividade de setup.

\section{Análise dos resultados}

Todos os cenários desenvolvidos foram analisados e seus resultados foram comparados estatisticamente em relação à produção obtida no cenário atual a partir de um teste de hipóteses.

Inicialmente foram obtidos os resultados dos números de peças produzidas para 10 réplicas em cada cenário. O teste de normalidade foi então realizado, onde verificou-se que os quatro cenários desenvolvidos mais o cenário atual podem ser aproximados de uma distribuição normal (Figuras 7 e 8 ). 
Figura 7 - Teste de Normalidade (Setup em etapas e dedicado)

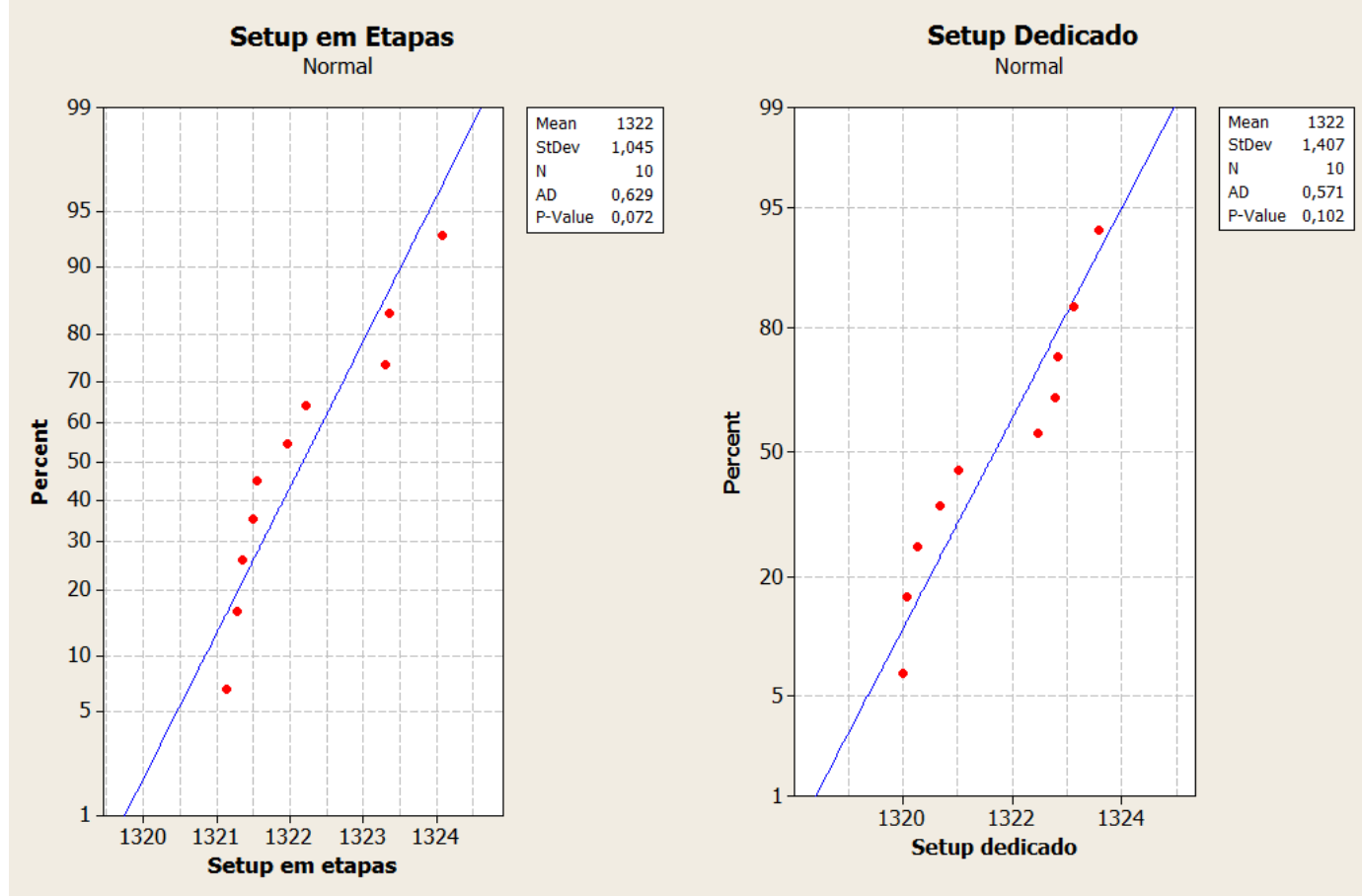

Fonte: Autoria própria (2010)

Figura 8 - Teste de Normalidade (Cenário 2, 3 e 4)
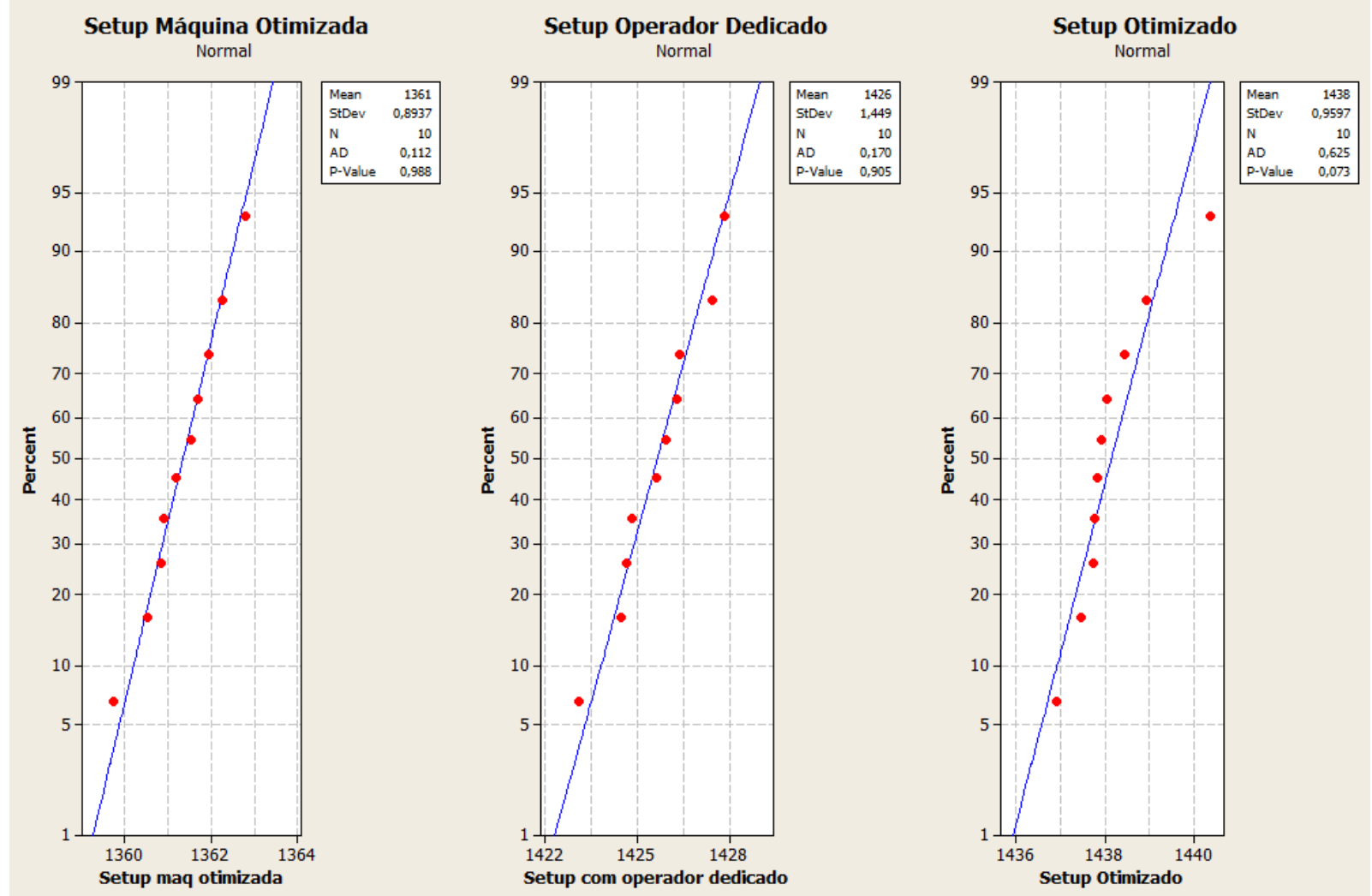

Fonte: Autoria própria (2010)

Como todos os p-values do testes de normalidade foram superiores ao nível de significância $(0,05)$, indicando que os dados poderiam ser aproximados de uma distribuição normal, realizou-se e o Teste f para os resultados de todos os cenários. Verificou-se que a variância dos dados dos 
cenários desenvolvidos quando comparados ao cenário atual são diferentes ( $p$-values $=0)$, a exceção do cenário 1 cujas variâncias são iguais (Figura 9).

Figura 9 - Teste de Variâncias (Teste f)



Fonte: Autoria própria (2010)

Por fim o teste $t$ foi realizado para verificar se as médias dos cenários gerados eram iguais as do cenário atual. De acordo com o teste de hipóteses realizado entre o cenário atual e o cenário 1 verificou-se que as médias dos valores obtidos são iguais, uma vez que seu $p$-value $(0,107)$ é superior ao nível de significância adotado. Desta forma não há alteração no número de peças produzidas. Ou seja, a forma como o setup será realizado (de forma dedicada ou em etapas) não influenciará no número de peças produzidas. O teste de hipóteses realizado com auxílio do software Minitab ${ }^{\circledR}$, os parâmetros do teste, bem como os resultados podem ser visualizados nas tabelas 6 e 7 .

Tabela 6 - Parâmetros do teste de hipóteses Two-Sample T

\begin{tabular}{ccccc}
\hline Teste & Tamanho da Amostra & Média & Desvio Padrão & Erro da média \\
\hline Setup em etapas & 11 & 1319,62 & 3,82 & 1,2 \\
Setup dedicado & 11 & 1316,16 & 5,61 & 1,7 \\
\hline
\end{tabular}

Fonte: Autoria própria (2010)

Tabela 7 - Resultado do teste de hipóteses Two-Sample T

\begin{tabular}{ccc}
\hline T-Value & P-Value & Desvio padrão Combinado \\
\hline 1,69 & 0,107 & 4,801 \\
\hline
\end{tabular}

Fonte: Autoria própria (2010) 
Os demais cenários apresentaram médias diferentes de acordo com os testes de hipóteses realizados. Quando a hipótese alternativa foi alterada para média maior que a do cenário atual, todos os p-values foram inferiores a 0,05, ou seja, as médias dos cenários 2, 3 e 4 são maiores que a do cenário atual, visto que nesse caso aceita-se a hipótese alternativa.

A figura 10 abaixo foi construída levando-se em consideração o aumento percentual médio em produtividade de cada cenário em relação ao cenário atual. A otimização das máquinas mostrouse estatisticamente significativa para o aumento de produtividade da célula, resultando em um acréscimo em produção da ordem de 3\%. Já a inclusão de um operador dedicado para a realização da atividade de setup contribuiu para um aumento médio de produção em torno de 7,8\%. Ainda em relação à figura 10 nota-se que em um cenário ótimo, onde o setup é realizado de forma dedicada, com a máquina otimizada e com inclusão de um novo operador pode alcançar um aumento de produtividade médio na ordem de 8,7\%. Apesar da figura 10 apresentar uma redução de produção da ordem de $0,04 \%$ para o cenário 1 quando comparado com o cenário atual, foi demonstrado que ambos são estaticamente iguais.

Vale ressaltar que a exceção do cenário 1, onde não existe nenhum tipo de investimento necessário, a empresa deve desenvolver um estudo relativo à viabilidade econômica para as mudanças propostas, visto que tal consideração foge aos objetivos inicias desse estudo.

Figura 10 - Percentual de aumento de produtividade por cenário em comparação com o cenário atual

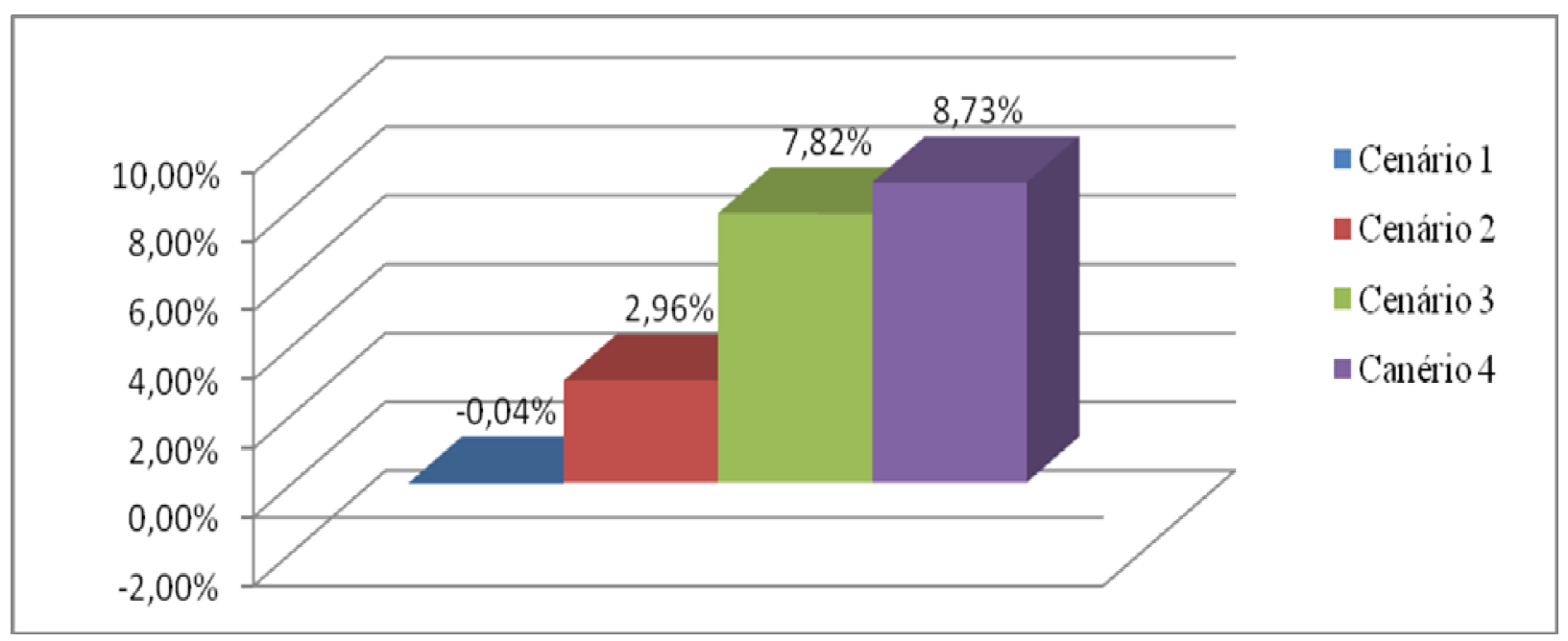

Fonte: Autoria própria (2010)

\section{Conclusão}

O projeto de simulação desenvolvido possibilitou afirmar para a empresa que independentemente da forma como o setup da linha de produção é realizado, seja de forma dedicada ou em etapas, a produção da célula será a mesma. 
Isso serviu para quebrar um paradigma na empresa, visto que a realização do setup de forma dedicada leva a uma falsa impressão de queda de produtividade, pois aos olhos dos gestores a presença de máquinas paradas quando da realização do setup em outra máquina, comprometeria a produção final, quando foi demonstrado que isso não a afetaria.

Este trabalho também auxiliou para melhorar as condições de trabalho do operador da célula, uma vez que a execução do setup de forma dedicada é mais prático de ser realizado do que o em etapas. Modo como vinha sendo feito.

De posse dessa informação três cenários foram desenvolvidos, visando à redução do tempo de setup e aumento de produtividade na célula. Pode-se verificar um aumento percentual em produtividade em todos os cenários em relação ao cenário um (setup dedicado), sendo que o ganho percentual mais significativo pode ser observado no cenário quatro (setup dedicado, com máquinas otimizadas e operador especializado na realização do setup), o qual apresentou um aumento de produtividade média na ordem de $8,73 \%$.

Cabe ressaltar que a escolha dos cenários simulados foi feita sob orientação da empresa em estudo, a qual tinha interesse em operar sua célula da maneira mais produtiva possível e reduzir ao máximo os tempos de setup. No entanto no desenvolvimento deste trabalho não foram considerados os custos envolvidos na implementação de cada cenário contrapondo aos ganhos alcançados em produtividade. Tal estudo ficou a cargo da empresa devido à confidencialidade dos dados que dessa forma complementará este trabalho internamente.

\begin{abstract}
Manufacturing environments have become increasingly complex, for this reason the decision-making processes are gaining complexity in the same proportion. In this context computer simulation has proved a valuable tool to assist in process improvement and analysis of changes. Through a computer simulation project in an auto parts supplier manufacturing cell, this paper aims to determine the best way to run setup: dedicated or in stages. In the end of the research it was possible to conclude that regardless of how the setup was accomplished, dedicated or in stages, the cell's average output was statistically equal. Because the dedicated setup was easier to be executed, three other scenarios were created from this one aiming to optimize the setup operation and increase the cell's productivity. All scenarios were built according to the company`s decision makers indication; this way, they could choose what scenario would better serve their needs.
\end{abstract}

Key-words: Computer simulation; setup; manufacturing.

\title{
Referências
}

ABDULMALEK, F. A.; RAJGOPAL, J. Analyzing the benefits of lean manufacturing and value stream mapping via simulation: A process sector case study. International Journal of Production Economics, 107, p. 223-236, 2007.

cross ${ }^{\text {ref }}$

AHMED, M. A.; ALKHAMIS, T. M. Simulation optimization for an emergency department healthcare unit in Kuwait. European Journal of Operation Research, p. 936-942. 2009.

ALlAHVERDI, A.; GUPTA, J. N. D.; ALDOWAISAN, T. A review of scheduling involving setup considerations. Omega, v. 27, p. 219-239, 1999. 
BANKS, J. Handbook of simulation: principles, methodology, advances, applications, and practice. Ed. John Wiley \& Sons, Inc., 864p. 1998.

cross ref

BANKS, J.; CARSON, J. S.; NELSON, B. L.; NICOL, D. M. Discrete-event system simulation. $2^{\text {a }}$ Edição. New Jersey: Prentice Hall, 2005.

BARROS, A. D. Algoritmo metaheurístico para busca do gargalo flutuante em flow shop permutacional com tempos de setup assimétricos e dependentes da seqüência. 2002. Dissertação (Mestrado) - Escola de Engenharia de São Carlos, USP, São Carlos.

BARROS, A. D.; MOCCELIN, J. V. Análise da flutuação do gargalo em flow shop permutacional com tempos de setup assimétricos e dependentes da seqüência. Gestão e Produção, v. 11, n. 1, p. 101-108, jan-abr, 2004.

cross ref

CHUNG, C. A. Simulation Modeling Handbook. A Practical Approach. CRC Press, London, New York. 2004.

CHWIF, L. Redução de modelos de simulação de eventos discretos na sua concepção: uma abordagem causal. $151 \mathrm{f}$. Tese (Doutorado em Engenharia Mecânica) - Escola Politécnica, Universidade de São Paulo, São Paulo, 1999.

CHWIF, L.; MEDINA, A. C. Modelagem e simulação de eventos discretos: teoria e aplicações. $2^{\mathrm{a}}$ Edição. São Paulo, Editora dos Autores, 2007.

EKREN, B. Y.; HERAGU, S. S.; KRISHNMURTHTY, A.; MALMBORG, C. J. Simulation based experimental design to identify factors affecting performance of AVS/RS. Computers \& Industrial Engineering, n.58, p.175-185, 2010.

FITZSIMMONS, J. A.; FITZSIMMONS, M. J. Administração de Serviços: operações, estratégia e tecnologia da informação. Porto alegre: 2a ed., Bookman, RS, 2000.

HARMON, R. L.; PETERSON, L. D. Reinventando a fábrica: conceitos modernos de produtividade aplicados na prática. Rio de Janeiro: Campus, 1991.

HARREL, C.; GHOSH, B. K.; BOWDEN, R. O. Simulation using promodel. 2. ed. New York: McGraw-Hill, 2004.

KELTON, W. D. Designing simulation experiments. In. WINTER SIMULATION CONFERENCE, 1999, Phoenix, AZ, USA. Proceedings... Phoenix, AZ, USA, 1999.

KIM, S. C.; BOBROWSKI, P. M. Impact of sequence-dependent setup time on job shop scheduling performance. International Journal of Production Research, v. 32, n. 7, p. 1503-1520, 1994.

cross'

LAM, K.; LAU, R. S. M. A simulation approach to restructuring call centers. Business Process Management Journal, Vol. 10, No. 4, P. 481-494. 2004.

cross ${ }^{\text {ref }}$

LAW, A. M. How to build valid and credible simulation models. In. WINTER SIMULATION CONFERENCE, 2006, Monterey, CA, USA. Proceedings... Monterey, CA, USA, 2006.

. How to build valid and credible simulation models. In: WINTER SIMULATION CONFERENCE, 2009, Austin, TX, USA. Proceedings... Austin, TX, USA, 2009.

LAW, A. M.; KELTON, W. D. Simulation modeling and analysis. 3.ed. New York: McGraw-Hill, 760 p. 2000.

MONTEVECHI, J. A. B.; LEAL, F.; PINHO, A. F.; COSTA, R. F. S.; OlIVEIRA, M. L. M.; SILVA, A. L. F. Conceptual modeling in simulation projects by mean adapted IDEF: an application in a Brazilian tech company. In. WINTER SIMULATION CONFERENCE, 2010, Baltimore, MD, EUA. Anais... Baltimore, MD, EUA, 2010.

O'KANE, J. F.; SPENCELEY, J. R.; TAYLOR, R. Simulation as an essential tool for advanced manufacturing technology problems. Journal of Materials Processing Technology, 107, p. 412-424, 2000. 
OliVEIRA, E. S.; SILVA, C. E. S.; MEllO, C. H. P.; SAlGADO, E. G.; CARDOSO, S. S. Análise da contribuição da simulação computacional no projeto para montagem. In. SIMPÓSIO DE ENGENHARIA DE PRODUÇÃO, XIII, 2006, Bauru. Anais... Bauru: SIMPEP, 2006.

PAIVA, C. N.; MONTEVECHI, J. A. B.; COSTA, R. F. S.; LEAL, F.; JESUS, J. T. Consideração de tolerâncias no tempo do trabalho humano em um modelo de simulação computacional. In. XLI SBPO, 2009, Porto Seguro. Anais... Porto Seguro: SBPO, 2009.

RYAN, J.; HEAVEY, C. Process modeling for simulation. Computers in Industry, v.57, p. 437-450, 2006.

SANCHEZ, S. M. Work smarter, not harder: guidelines for designing simulation experiments. In. WINTER SIMULATION CONFERENCE, 2007, Washington. Proceedings... Washington, DC, USA, 2007.

SARGENT, R.G. Validation and verification of simulation models. In. WINTER SIMULATIONS CONFERENCE, 2008, Miami. Proceedings... Miami, FL, USA, 2008.

SLACK, N.; CHAMBERS, S.; JOHNSTON, R. Administração da produção. 2. ed. São Paulo: Atlas, 2002.

TRIOLA, M. F. Introdução a Estatística. 9a Edição, Editora LTC, 2005.

\section{Agradecimentos}

Os autores agradecem à FAPEMIG, CNPq, CAPES e PADTEC pelo suporte financeiro ao presente trabalho.

\section{Dados do autor:}

Nome completo: Rafael de Carvalho Miranda

Filiação institucional: Universidade Federal de Itajubá - UNIFEI

Departamento: Instituto de Engenharia de Produção e Gestão - IEPG

Função ou cargo ocupado: Mestrando Engenharia de Produção

Endereço para correspondência (bairro, cidade, estado, país e CEP): Rua Luiz Gama, 53, bairro Varginha, Itajubá - MG. CEP: 37501128.

Telefones para contato: (35) 9962-4694

e-mail: mirandaprod@yahoo.com.br

\section{Nome completo: Bárbara Coutinho Fernandes}

Filiação institucional: Universidade Federal de Itajubá - UNIFEI

Departamento: Instituto de Engenharia de Produção e Gestão - IEPG

Função ou cargo ocupado: Mestranda Engenharia de Produção

Endereço para correspondência (bairro, cidade, estado, país e CEP): Rua Cristina, 112, Bairro Boa Vista, Itajubá - MG. CEP: 37505072.

Telefones para contato: (35) 9168-3221

e-mail: babifernan@yahoo.com.br 
Nome completo: Janaína Rodrigues Ribeiro

Filiação institucional: Universidade Federal de Itajubá - UNIFEI

Departamento: Instituto de Engenharia de Produção e Gestão - IEPG

Função ou cargo ocupado: Mestranda Engenharia de Produção

Endereço para correspondência (bairro, cidade, estado, país e CEP): Rua José Manso Pereira

Cabral, 146, Bairro São Vicente, Itajubá - MG. CEP: 37502038.

Telefones para contato: (35) 8819-7999

e-mail: janaina_epr@yahoo.com.br

Nome completo: José Arnaldo Barra Montevechi

Filiação institucional: Universidade Federal de Itajubá - UNIFEI

Departamento: Instituto de Engenharia de Produção e Gestão - IEPG

Função ou cargo ocupado: Professor Titular

Endereço para correspondência (bairro, cidade, estado, país e CEP): Av. BPS, 1303, bairro

Pinheirinho, Itajubá - MG. Caixa Postal 50. CEP: 37500903.

Telefones para contato: (35) 3629-1290

e-mail: montevechi@unifei.com.br

\section{Nome completo: Alexandre Ferreira de Pinho}

Filiação institucional: Universidade Federal de Itajubá - UNIFEI

Departamento: Instituto de Engenharia de Produção e Gestão - IEPG

Função ou cargo ocupado: Professor Adjunto

Endereço para correspondência (bairro, cidade, estado, país e CEP): Av. BPS, 1303, bairro

Pinheirinho, Itajubá - MG. Caixa Postal 50. CEP: 37500903.

Telefones para contato: (35) 3629-1307

e-mail:pinho@unifei.com.br

Recebido para publicação em: 12/08/2010

Aceito para publicação em: 08/10/2010 